\title{
Stable Optical Vortex Solitons in Pair Plasmas
}

\author{
V. I. Berezhiani \\ Andronikashvili Institute of Physics, Tbilisi 0177, Georgia \\ S. M. Mahajan* \\ Institute for Fusion Studies, The University of Texas at Austin, Austin, Tx 78712 \\ N. L. Shatashvilit \\ Faculty of Exact and Natural Sciences, \\ Javakhishvili Tbilisi State University, Tbilisi 0128, Georgia \\ Andronikashvili Institute of Physics, Tbilisi 0177, Georgia
}

\begin{abstract}
It is shown that the pair plasmas with small temperature asymmetry can support existence of localized as well as de-localized optical vortex solitons. Coexistence of such solitons is possible due to peculiar form of saturating nonlinearity which has a focusing-defocusing nature - for weak amplitudes being focusing becoming defocusing for higher amplitudes. It is shown that delocalized vortex soliton is stable in entire region of its existence while single- and multi-charged localized vortex solitons are unstable for low amplitudes and become stable for relativistic amplitudes.
\end{abstract}

PACS numbers: 52.27.Cm, 52.27.Ep, 52.30.Ex, 52.35.Hr, 52.35.Mw, 52.35.Sb, 42.65.Tg

${ }^{*}$ Electronic address: mahajan@mail.utexas.edu

${ }^{\dagger}$ Electronic address: shatash@ictp.it 


\section{MODEL}

The richness of an electromagnetically active medium is often measured by the variety of structures that it can support. Such structures, in turn, are created because of the nonlinear response of the medium, for instance, to the impact of a large amplitude electromagnetic wave. Naturally the properties of the structure (its shape, its content, its stability, its angular momentum etc.) are dictated by the type of nonlinearity that can arise in the medium. The discovery or identification of a new nonlinearity type, then, opens up a new era of investigation, even, discovery.

In this paper we work out some of the consequences of a new focusing-defocusing nonlinearity [1] belonging to the general class of saturating nonlinearities (whose magnitude tends to a constant as the wave amplitude becomes large). Saturating nonlinearities seem to appear, inter alia, in theories of large amplitude wave propagation in pair plasmas (plasmas whose main constituents have equal mass and opposite charge [2, 3, 4] ) in which the pair symmetry is broken by some physical mechanism. For instance, a small amount of Baryonic matter (protons) may break the symmetry of an electron-positron (e-p) plasma in the MEV era of the early universe [5, 6, 7, 8]. In recently created pair ion (PI) plasmas in the laboratory, a variety of symmetry breaking mechanisms like the small contamination by a much heavier immobile ion, or a small mass difference between the two constituent species, could exist [9, 10, 11, 12, 13]. Asymmetries originating in small temperature differences in the constituent species may be always available for structure formation: in the laboratory such a temperature difference could be readily engineered (in a controlled way) and there are reasons to believe that species temperature difference could exist in cosmic and astrophysical setting where one encounters e-p plasmas. It is in this latter setting that a new type of nonlinearity

$$
F\left(|A|^{2}\right)=\frac{\epsilon^{2}}{2} \frac{\kappa|A|^{2}}{\left(1+\kappa|A|^{2}\right)^{2}}
$$

appeared while deriving the wave equation (in parabolic approximation) [1]

$$
2 i \omega_{0} \frac{\partial A}{\partial t}+\frac{(2-\epsilon)}{\omega_{0}^{2}} \frac{\partial^{2} A}{\partial \xi^{2}}+\nabla_{\perp}^{2} A+F\left(|A|^{2}\right) \cdot A=0
$$

describing the nonlinear evolution of the vector potential of an electromagnetic pulse propagating in an arbitrary pair plasma with temperature asymmetry. Following assumptions and notations are necessary in order to put equations (1) and (2) in perspective: $A$ is the slowly 
varying amplitude of the circularly polarized EM pulse $\sim A(\hat{\mathbf{x}}+\hat{\mathbf{y}}) \exp \left(\mathrm{ik}_{0} \mathrm{z}-\omega_{0} \mathrm{t}\right)$ with mean frequency $\omega_{0}$ and mean wave number $k_{0} ; \nabla_{\perp}^{2}=\partial^{2} / \partial x^{2}+\partial^{2} / \partial y^{2}$ is the diffraction operator and $\xi=z-v_{g} t$ is the "comoving" (with group velocity $v_{g}$ ) coordinate.

Equation (22) is written in terms of the dimensionless quantities $A=|e| A /\left(m G\left(T_{0}^{-}\right) c^{2}\right)$, $r=\left(\omega_{e} / c\right) r, \quad t=\omega_{e} t$; where $\omega_{e}=\left(4 \pi e^{2} n_{0} / m\right)^{1 / 2}$ is the electron Langmuir frequency and $m$ is the electron mass. The charges $q^{ \pm}$and masses $m^{ \pm}$of positive and negative ions are assumed to be same (in this paper we mainly concentrate on the specific case of pair plasma consisting of electrons and positrons, i.e. $q^{+}=e^{+}=q^{-}=-e^{-}=|e|$ and $m^{+}=m^{-}=m$ ). The equilibrium state of the system is characterized by an overall charge neutrality $n_{0}^{+}=$ $n_{0}^{-}=n_{0}$ where $n_{0}^{+}$and $n_{0}^{-}$are the unperturbed number densities of the positive and negative ions respectively. The background temperatures of plasma species are $T_{0}^{ \pm}\left(T_{0}^{+} \neq T_{0}^{-}\right)$ and $m G\left(z^{ \pm}\right)=m K_{3}\left(z^{ \pm}\right) / K_{2}\left(z^{ \pm}\right)$is the "effective mass", $\left[z^{ \pm}=m c^{2} / T^{ \pm}\right]$, where $K_{\nu}$ are the modified Bessel functions. For the nonrelativistic temperatures $\left(T^{ \pm} \ll m c^{2}\right) G^{ \pm}=$ $1+5 T^{ \pm} / 2 m c^{2}$ and for the ultra-relativistic temperatures $\left(T^{ \pm} \gg m_{\alpha} c^{2}\right) G^{ \pm}=4 T^{ \pm} / m c^{2} \gg 1$. The smallness parameter $\epsilon=\left[G\left(T_{0}^{+}\right)-G\left(T_{0}^{-}\right)\right] / G\left(T_{0}^{+}\right)$measures the temperature asymmetry of plasma species. For the nonrelativistic temperatures $\epsilon=5\left(T_{0}^{+}-T_{0}^{-}\right) / 2 m c^{2}$ while in ultrarelativistic case $\epsilon=\left(T_{0}^{+}-T_{0}^{-}\right) / T_{0}^{+}$. The numerical factor $\kappa=1 / 2$ for non-relativistic temperatures ( $=2 / 3$ for ultrarelativistic temperatures). In deriving Eq.(2) with (11), we have assumed that the plasma is transparent $\left(\omega_{0} \gg 1, v_{g} \simeq 1\right)$, and that the longitudinal extent of the pulse is much shorter than its transverse dimensions. However, despite of $\partial A / \partial \xi \gg \nabla_{\perp} A$, the second and the third terms in Eq.(2) can be comparable due to the transparency of the plasma $\left(\omega_{0} \gg 1\right)$.

With self-evident renormalization the equation (2) can be written as:

$$
i \frac{\partial A}{\partial t}+\frac{\partial^{2} A}{\partial \xi^{2}}+\nabla_{\perp}^{2} A+f\left(|A|^{2}\right) \cdot A=0,
$$

where the nonlinearity function is now following [1]:

$$
f\left(|A|^{2}\right)=\frac{|A|^{2}}{\left(1+|A|^{2}\right)^{2}} .
$$

which has an unusual feature - in the ultrarelativistic limit $\left(|A|^{2} \gg 1\right)$ it tends to be 0 .

Note that the nonlinear refraction index for the considered system can be written as $\delta n_{n l}=f(I)$, where $I=|A|^{2}$ is the intensity of the EM field. The medium is a self-focusing $\left(d\left(\delta n_{n l}\right) / d I>0\right)$ provided $I<1$ while for higher intensities $(I>1)$, it becomes defocusing 
$\left(d\left(\delta n_{n l}\right) / d I<0\right)$. For the localized intense EM pulse with the peak intensity $I_{m}>1$ the medium becomes defocusing at the peak while remaining focusing at the wings of the EM pulse intensity profile.

In [1] we have demonstrated that Eq.(3) supports existence of the stable solitonic structures for any spatial dimensions $(D=1,2,3)$. Such "light-bullets" exist provided that the amplitude of the solitons is lower than certain critical values (for instance, in 1-dimensional $(1 D)$ media $\left.A_{m}<A_{m c r} \simeq 1.4\right)$. It is important to emphasize that at $A_{m} \rightarrow A_{m c r}$ the profile of the central part of the soliton flattens and widens at the top. The existence of flat-top soliton can be explained by the peculiarities of our focusing-defocusing nonlinearity implying that the pulse top part with $A>1$ entered the defocusing region having the tendency of diffraction while the wings of the soliton are in focusing region preventing the total spread of the pulse.

\section{FORMATION OF VORTICES}

In this section we examine the possibility of the formation of two-dimensional stable soliton-structures carrying a screw type of dislocation, i.e., optical vortices. The generation, propagation, and interaction of optical vortices in nonlinear media has been a subject of extensive studies (see for review [14]). In a self-defocusing medium an optical vortex soliton (OVS) is $(2+1)$-dimensional (two transverse dimension and a time) stationary beam structure with phase singularity. An OVS is a dark spot, i.e., a zero intensity center surrounded by a bright infinite background. Self-focusing media also support localized optical vortex soliton solutions (LOVS) with phase dislocation surrounded by the bright ring. In self-focusing medium LOVS are unstable against symmetry breaking perturbations that lead to the breakup of rings into filaments [15].

Our nonlinearity (4) has focusing-defocusing features, hence, one could expect that formation of both OVS and LOVS solutions is possible in the medium. Such statement can be augmented by the results of [16] where focusing-defocusing model of the media was postulated to be cubic-quintic medium with sign-changing nonlinearity $\left(f\left(|A|^{2}\right)=|A|^{2}-|A|^{4}\right)$. In contrast to the cubic-quintic models the saturation nonlinearity derived by us in [1] is not sign-changing - it is the focusing-defocusing one. To verify this expectation we assume that the pulse is sufficiently long and effects related to the group velocity dispersion 
$\left(\sim \omega_{0}^{-2} \partial^{2} A / \partial \xi^{2}\right)$ can be ignored.

Introducing polar coordinates $(r, \theta)$ in $(x, y)$ plane, we look for solutions of Eq.(3i) in the form of

$$
A=A(r) \exp (i \lambda t+i m \theta),
$$

where integer $m$ defines the topological charge of vortices and $\lambda$ is the nonlinear frequency shift. The ansatz (5) converts Eq. (3) to the ordinary differential equation

$$
\frac{d^{2} A}{d r^{2}}+\frac{1}{r} \frac{d A}{d r}-\frac{m^{2}}{r^{2}} A-\lambda A+\frac{A^{3}}{\left(1+A^{2}\right)^{2}}=0 .
$$

We have used numerical methods to find the localized solutions of (66). It is possible to map the equation in the $\left(A, A_{r}\right)$ plane (phase plane) and show that it admits both OVS and LOVS solutions. LOVS can exist in the form of infinite number of discrete bound states with $A_{n}(r)(n=1,2, \ldots)$ where $n$ denotes the finite $r$ zeros of the eigenfunction.

In what follows we consider only the lowest order (lowest radial eigenmode) solution of Eq.(6) $(n=1)$. For nonzero $m$ (the case we are interested in), the ground state LOVS is positive, has a node at the origin $r=0$, reaching a maximum, and then monotonically decreases with increasing $r$. Such localized solution exists if $\lambda>0$ with the following asymptotic behavior: $\quad A_{r \rightarrow 0} \rightarrow r^{|m|} A_{0}$ and $A_{r \rightarrow \infty} \rightarrow \exp (-r \sqrt{\lambda}) / \sqrt{r}$, where $A_{0}$ is a constant which measures the slope of $A$ at origin. OVS solutions have the same asymptote for $r \rightarrow 0$, while for $r \rightarrow \infty$ the amplitude has a nonzero value $A(r)=A_{\infty}+m^{2} /\left(r^{2} f^{\prime}\left(A_{\infty}\right)\right)$ . Here, $\lambda=f\left(A_{\infty}\right)$ and OVS exists when $f^{\prime}\left(A_{\infty}\right)<0$, i.e. when background intensity of the soliton (far beyond the vortex core) is relativistic $A_{\infty}>1$. In dimensional units this condition corresponds to the negative slope of the nonlinear refractive index $\left(d \delta n_{n l} / d I<\right.$ $0)$, i.e., in the asymptotic region of the solution the medium is defocusing. It is easy to demonstrate [1] that the constant background field with $A_{\infty}>1$ is modulationally stable.

A shooting code was used to numerically solve Eq.(6). To better understand the results of simulations we use the analogy with a nonconservative motion of a particle. For this purpose one can rewrite the Eq.(6) as

$$
\frac{d}{d r}\left[\left(\frac{d A}{d r}\right)^{2}+V(A)\right]=\frac{m^{2}}{r^{2}} \frac{d A^{2}}{d r}-\frac{2}{r}\left(\frac{d A}{d r}\right)^{2},
$$

where the "effective potential" is $V(A)=-\lambda A^{2}+\ln \left(1+A^{2}\right)-A^{2} /\left(1+A^{2}\right)$. The profile of the potential $V(A)$ for different values of $\lambda$ is presented in Fig.1. The potential has 
the maxima at the points $A=0$ and $A_{\max }=\sqrt{[1-2 \lambda+\sqrt{1-4 \lambda}] / 2 \lambda}$. The bounded solution is possible only in the case $0<\lambda<0.25$ while $A_{\max }>1$.

The OVS solutions correspond to a particle beginning its motion at origin $(A=0)$ with certain initial $A_{0}$ (which can be termed as a velocity (if $m=1$ ) or acceleration (if $m=2$ and so on) and dissipating its initial energy approaching asymptotically potential maximum at $A_{\text {max }}$. Thus, the background intensity of OVS is $A_{\infty}=A_{\max }$, it is always larger than unity and can become arbitrarily large for $\lambda \rightarrow 0$. We also found out that OVS solutions exist even for $0.25>\lambda>\lambda_{c r} \simeq 0.2162$, i.e., when $V\left(A_{\max }\right)<0$ (see curve "a" in Fig.1). In other words, the effective particle cannot overpass but only approach asymptotically the lower potential maximum.

The numerical solutions of the nonlinear equation (66) for $m=1,2$ and 3 are shown in Fig.2. As expected, the soliton-like solutions evidently go to zero as $r^{m}$ for small $r$, and reach an $m$-independent asymptotic value predicted above. In Fig.3 curve "a" displays the dependence of the field derivative at the origin, $A_{0}$, as a function of the nonlinear frequency shift $\lambda$ for $m=1$ case. One can see that $A_{0}$ is a growing function for small $\lambda$-s . For small $\lambda$-s the position of the potential maximum "moves" to larger values of $A$ and, consequently, "particle" needs to have larger initial "velocity" $\left(A_{0}\right)$ to reach the maximum.

In contrast to OVS, the LOVS solutions correspond to the particle returning back asymptotically to the initial position at $A=0$. It seems obvious that due to the "frictional" motion particle can not make its way back if $\lambda>\lambda_{c r}$. Thus, LOVS may exist in the range $0<\lambda<\lambda_{c r}$ while its amplitude (in contrast to OVS) is a growing function of $\lambda$. Such dependence is obtained numerically and is presented in Fig.4. for single-charged vortices $(m=1)$. One can see that the amplitude of the LOVS $\left(A_{m}\right)$ is bounded from above by certain critical value for $A_{c r}(\simeq 1.5)$. Thus, in contrast to OVS the localized vortex can be just moderately relativistic. Notice that for $0.16 \leq \lambda \leq \lambda_{c r}$ the amplitude of the LOVS $\left(A_{m}\right)$ varies in the range $1 \leq A_{m} \leq A_{c r}$. For the top part of such solution (with $A(r)>1$ ) the medium is defocusing while remains focusing for lower intensity wings of the structure. Consequences of this fact can be seen in Fig.5 where profile of LOVS is given for variety of $\lambda$. With increase of $\lambda$ the central part of the LOVS flattens and widens converging to the OVS. In principle, it is possible to create flat-top LOVS with a large transverse width. Convergence of LOVS to OVS can be better seen in Fig.3 where the curve "b" corresponding 
to LOVS almost coincides with curve "a" near the point $\lambda \approx \lambda_{c r}$. Similar behavior of the solutions can be obtained for the vortices with higher charge $(m=2,3, .$.$) , however, the$ corresponding figures we do not present here for brevity.

\section{STABILITY OF SOLUTIONS}

Are these solitonlike solutions stable?

The intensity dependent switching from the focusing to defocusing regime can have an interesting consequence for the stability properties of the solutions. As it is well established [14] OVSs with $m=1$ are stable whereas vortices with a larger value of $m$ may decay into the single-charged ones in self-defocusing media. In our case the bulk of the OVS is always in the defocusing regime and as we mentioned above the background field is always stable. However, near to vortex core the medium becomes focusing. Thus, stability of the OVS in our case can not be granted.

We performed stability analysis solving numerically Eq.(3); while simulation (for various $\lambda$-s), initial stationary OVS state was perturbed radially and azimuthally by Gaussian noise. Typical picture of the evolution is plotted is Fig.6. We see that perturbations are quickly radiated away and the initial state relaxes to the ground state solution implying that the OVS is stable in the whole examined range.

To verify stability of LOVS we first performed a linear stability analysis. To do so we follow procedure developed by [17] and consider perturbation acting along a ring of mean radius $r_{*}$, where $A\left(r_{*}\right)=A_{m}$. Assuming constant intensity and spatial uniformity for this ring, one can rewrite the diffraction operator in (3) as $\nabla_{\perp}^{2}=r_{*}^{-2} \partial^{2} / \partial \theta^{2}$ and introducing azimuthal perturbation with a phase factor $\Psi=\Omega t+M \theta$ (where $M$ is an integer) for the growth rate of instability we get:

$$
\operatorname{Im}(\Omega)=\frac{M}{r_{*}} \operatorname{Re}\left[\frac{2\left(1-A_{m}^{2}\right)}{\left(1+A_{m}^{2}\right)^{3}}-\frac{M^{2}}{r_{*}^{2}}\right] .
$$

One can see from (8) that large amplitude LOVS with $A_{m}>1$ is always stable. For the lower amplitude case LOVS should decay into $M_{\max }$ multiple filaments, where $M_{\max }$ is an integer close to the number for which maximal growth rate is maximal.

In Fig.7 we plot $\operatorname{Im}(\Omega)$ versus $M$ for $\lambda=0.1$ and for $m=1,2,3$. Corresponding $A_{m}$ are respectively $0.66 ; 0.65 ; 0.63$ and $r_{*}=6.3 ; 11.6 ; 16.9$. One should expect that 
instability will result in breaking of the pulse into the filaments (fragments) with number of filaments being respectively 2,4 , and 5 (or 6 ) for $m=1,2,3$. These filaments have to conserve the total angular momentum. Since the fusion of filaments is not possible due to the topological reasons, they can eventually spiral about each other or fly off tangentially to the initial ring generating bright solitonic structures found for index saturation nonlinearity [15].

Our numerical simulations for $A_{m}<1$ give evidence of a quickly developing instability in agreement with predictions of linear stability analysis. Indeed, in Fig.8 we present the results of simulations for the LOVS with $m=1$ and 2 . The LOVS with $m=1$ breaks into two filaments while for $m=2$ the breaking into 4 filaments takes place. The filaments are running away tangentially without spiraling. All filaments like spatial solitons remain stable. Most interesting is the situation when amplitude of LOVS is larger than unity.

In Fig.9 we present the evolution of LOVS both for $m=1$ and $m=2$; corresponding amplitudes for the soliton solutions are $(\lambda=0.2): A_{m}=1.39$ and $A_{m}=1.37$, respectively. The initial input LOVS solution was modulated by a Gaussian noise (the level of noise was $5 \%$ ). One can see that breaking of the LOVS does not take place. In order to be sure that some very slow instability is not developing the simulations were carried out until $t=4000$, i.e., for 130 soliton period $T_{\text {sol }}=2 \pi / \lambda \approx 30$. Thus, single and multi-charged LOVS become stable for large amplitudes.

At the end we would like to emphasize that if for single-charged LOVS we are confident about its stability for multi-charge LOVS (as well as for OVS) one should be careful. Indeed, from general topological reasons the multi-charged vortices are supposed to be unstable and they should break into single charge vortices. However, we could not observe in our simulations such breaking. It is possible that this instability is very slow (subexponential/algebraic) and, as a result we obtained that multi-charged vortices are very long-lived objects and practically stable.

In our consideration the effects related to the group velocity dispersion and corresponding reshaping of the radiation have been ignored. However, one can generalize our results by keeping the term $\sim \partial^{2} A / \partial \xi^{2}$ in Eq.(3). In transparent plasma case this term can affect the long time dynamics of self-guiding vortex solitons. In particular due to weak modulation 
instability [18] the self-trapped beam eventually will break into a train of spatiotemporal solitons, i.e. the "light bullets". However, due to the topological reasons the vortex lines should survive the structural changes. We expect that instability will result in generation of fully localized bullets of vortex solitons (the spinning-bullets). Dynamics of formation and stability of such structures is beyond of the scope of current paper.

\section{CONCLUSIONS}

The asymmetries originating in small temperature differences in the constituent species of electromagnetically active medium may be always available for structure formation both in laboratory and cosmic/astrophysical settings. In present paper we have shown that this asymmetry defines the specific properties of the structure due to the new type of the nonlinearity that can arise in the medium (derived in our earlier paper [1]). We found that the pair plasmas of any dimension with "asymmetry" in initial temperatures can support the stable large amplitude optical vortex and localized vortex solitons. Localized structures for certain parameters may have the flat-top shapes. The coexistence of LOVS and OVS solutions and their stability in such medium is due to the specific form of saturating nonlinearity switching from the self-focusing to the self-defocusing regime and vice versa. This fact is rather interesting specifically for laboratory conditions.

$* * *$

Authors express their special thanks to Doctors S.I. Mikeladze and K.I. Sigua for the interest. The work of SMM was supported by USDOE Contract No. DE-FG 03-96ER54366. The work of NLS and VIB was supported by ISTC Project G-1366 and Georgian NSF grant projects GNSF 69/07 (GNSF/ST06/4-057) and GNSF 195/07 (GNSF/ST07/4191).

[1] S.M. Mahajan, N.L. Shatashvili and V.I. Berezhiani. Phys. Rev. E, submitted (2009); arXiv:0909.0587v1 [physics.plasma-ph] (2009).

[2] P.A. Sturrock, Astrophys. J. 164, 529 (1971); M.A. Ruderman, P.G. Sutherland, Astrophys. J. 196, 51 (1995); F.C. Michel, Theory of Neutron Star Magnetospheres, University of Chicago 
Press, Chicago, 1991.

[3] M.C. Begelman, R.D. Blandford, M.J. Rees, Rev. Mod. Phys. 56 (1984) 255.

[4] T. E. Cowan et al., Laser Part. Beams 17, 773 (1999); C. Gahn et al., Appl. Phys. Lett. 77, 2662 (2000); Chen et a., Phys. Rev. Lett. 102, 105001 (2009).

[5] S. Wineberg. Gravitation and Cosmology: Principles and Applications of the General Theory of Relatvity. John Wiley and Sons, Inc. 1972.

[6] M. E. Gedalin, J.G. Lominadze, L. Stenflo and V.N. Tsitovich, Astrophys. Space Sci. 108, 393 (1985); P.K. Shukla, N.N. Rao, M.Y. Yu and N.L. Tsintsadze. Phys. Reports. 131, 1 (1986).

[7] V.I. Berezhiani and S.M. Mahajan. Phys. Rev. Lett. 73, 1110 (1994); V.I. Berezhiani and S.M. Mahajan. Phys. Rev. E 52, 1968 (1995).

[8] T. Cattaert, I. Kourakis and P.K. Shukla. Phys. Plasmas, 12, 012310 (2005); P. K. Shukla and M. Khan, Phys. Plasmas 2, 014504 (2005); T. Tatsuno, M. Ohhashi V.I. Berezhiani and S.V. Mikeladze. Phys. Letters, A 363, 225 (2007).

[9] W. Oohara and R. Hatakeyama. Phys.Rev.Lett., 91, 205005 (2003); W. Oohara, D. Date, and R. Hatakeyama. Phys. Rev. Lett., 95, 175003 (2005).

[10] W. Oohara, Y. Kuwabara, and R. Hatakeyama. Phys. Rev. E., 75, 056403 (2007).

[11] W. Oohara and R. Hatakeyama. Phys. Plasmas, 14, 055704 (2007).

[12] S.M. Mahajan and N.L. Shatashvili. Phys. Plasmas. 15, 100701 (2008); A. Esfandyari-Kalejahi, I. Kourakis, M. Mehdipoor and P.K. Shukla. J. Phys. A: Math.Gen., 39, 13817 (2006).

[13] N.L. Shatashvili, J.I. Javakhishvili, and H. Kaya. Astrophys. Space Sci. 250, 109 (1997); N.L. Shatashvili and N. Rao. Phys. Plasmas, 6, 66 (1999).

[14] Desyatnikov, A., L. Torner, and Y. S. Kivshar, Prog. in Opt. 47, 291 (2005).

[15] W. J. Firth and D. V. Skryabin, Phys. Rev. Lett. 79, 2450 (1997); D. V. Skryabin and W. J. Firth, Phys. Rev. E 58, 3916 (1998).

[16] V. Skarka, V.I. Berezhiani and R. Miklaszewki. Phys. Rev.E 56, 1080 (1997); V.I. Berezhiani, V. Skarka and N.B. Aleksic. Phys. Rev. E 64, 057601(2001).

[17] j. Atai, Y. Chen, J.M. Soto-Crespo. Phys. Rev. A 49, R3170 (1994); Vicotte and L. Berge, Physica D: Nonlinear Phenomena 223, 163(2006).

[18] N.A. Akhmediev and J.M. Soto-Crespo, Phys. Rev. A 47, 1358 (1993); S.M. Mahajan, V.I. Berezhiani and R. Miklaszewki. Phys. Plasmas. 5, 3264 (1998). 




FIG. 1: The "effective potential" versus the amplitude for different values of the nonlinear frequency shift $\lambda$. The curve "a" corresponds to $\lambda>\lambda_{c r} \simeq 0.2162$, the curve "b" has $\lambda=\lambda_{c r}$, and for the curve "c" $0<\lambda<\lambda_{c r}$.

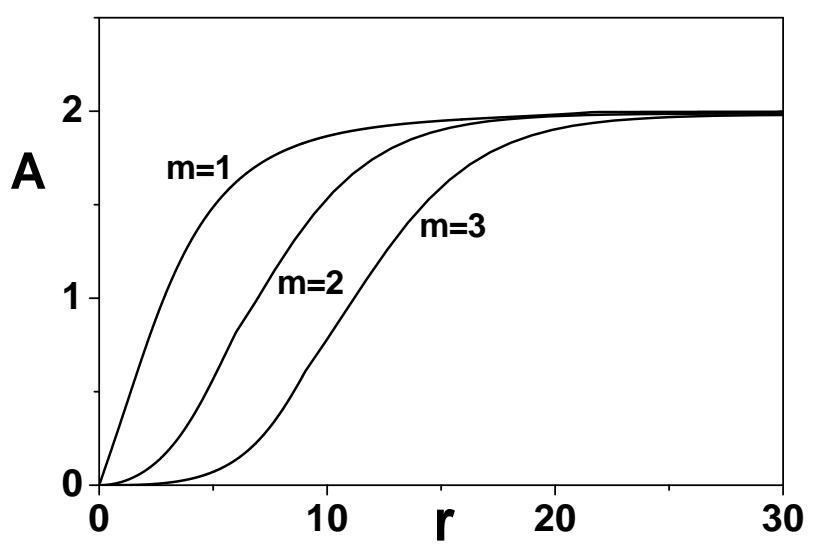

FIG. 2: Profiles of OVS-s for $m=1, m=2, m=3$; nonlinear frequency shift $\lambda=0.16$. 


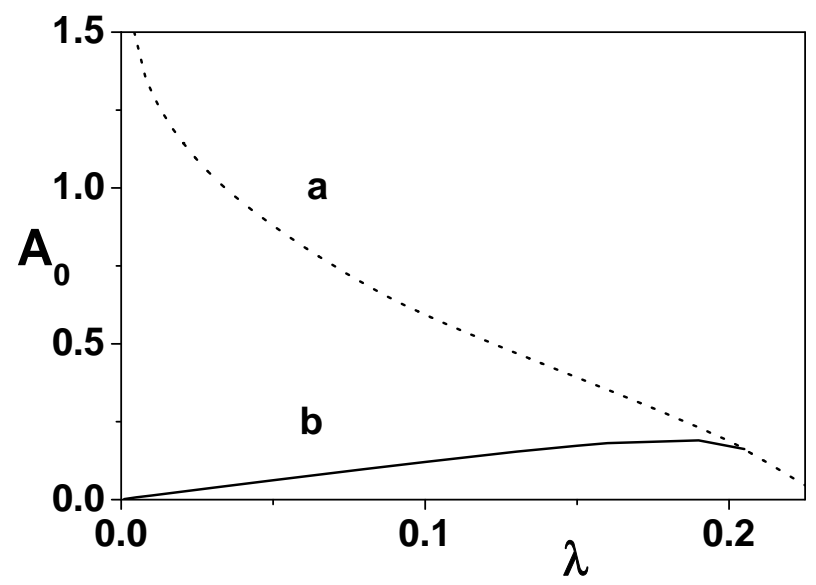

FIG. 3: $A_{0}$ versus $\lambda$ for $m=1$; curve "a" corresponds to OVS while curve "b" - to LOVS.

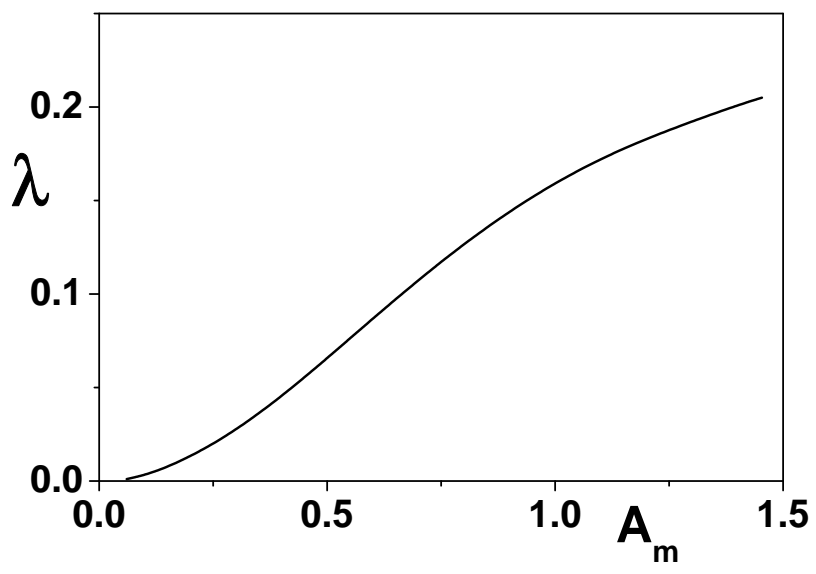

FIG. 4: The effective eigenvalue $\lambda$ versus soliton amplitude $A_{m}$ for $m=1$.

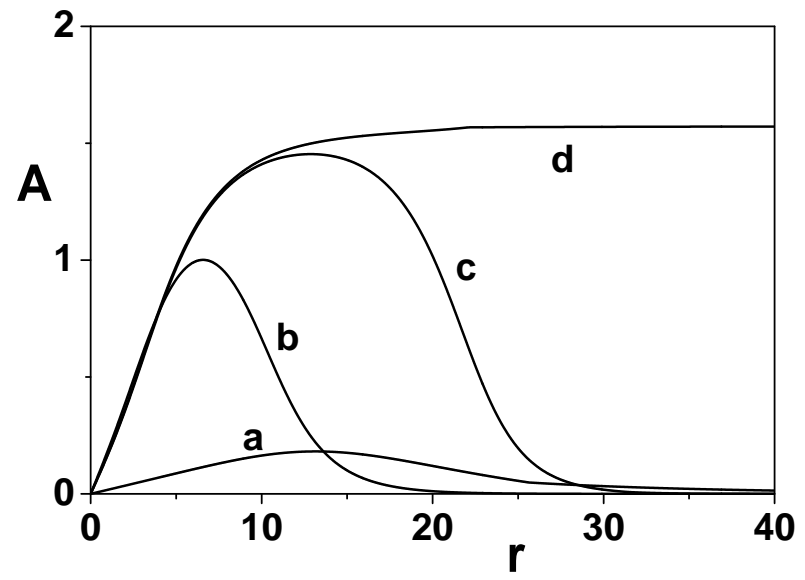

FIG. 5: Profiles of soliton solutions. Curves "a", "b" and "c" correspond to LOVS with $\lambda=$ $0.005 ; 0.16 ; 0.205$, respectively. Curve "d" corresponds to OVS for $\lambda=0.205$. 


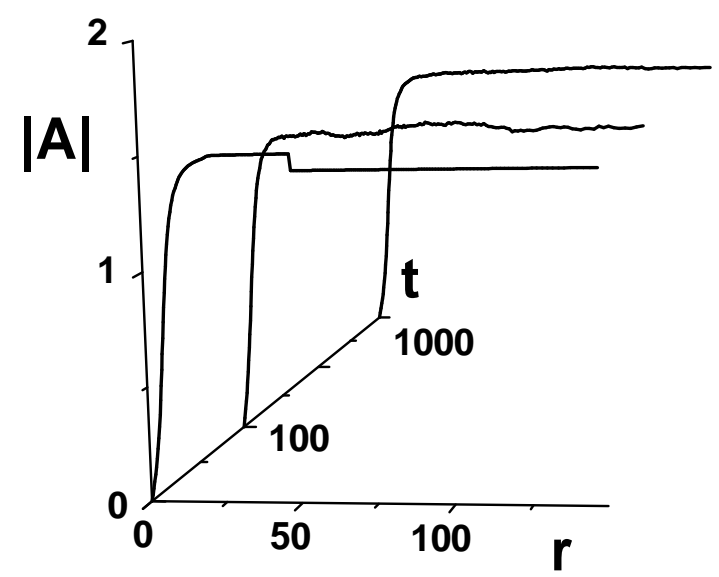

FIG. 6: The dynamics of initially perturbed OVS-s: plots are chosen for different time-moments $t=0 ; 100 ; 1000$.

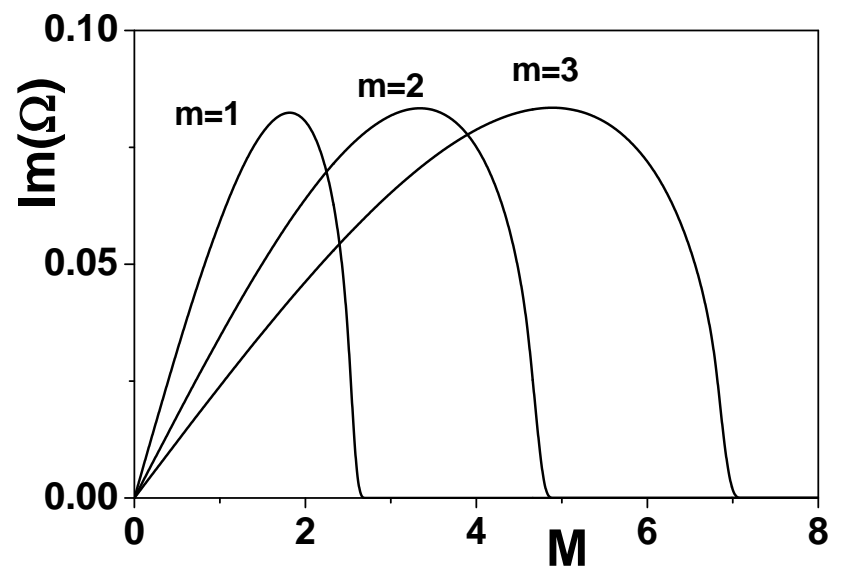

FIG. 7: Instability growth rate $\operatorname{Im}(\Omega)$ versus $M$ for $\lambda=0.1$ for different topological charges $m$. 




FIG. 8: Vortex dynamics (for different time-moments) when $\lambda=0.1$ : the left panel - for $m=$ 1, $A_{\max }=0.66$, the vortex splits into 2 filaments; the right panel - for $m=2, A_{\max }=0.6580$, the vortex splits into 4 filaments; the filaments are running away tangentially. 

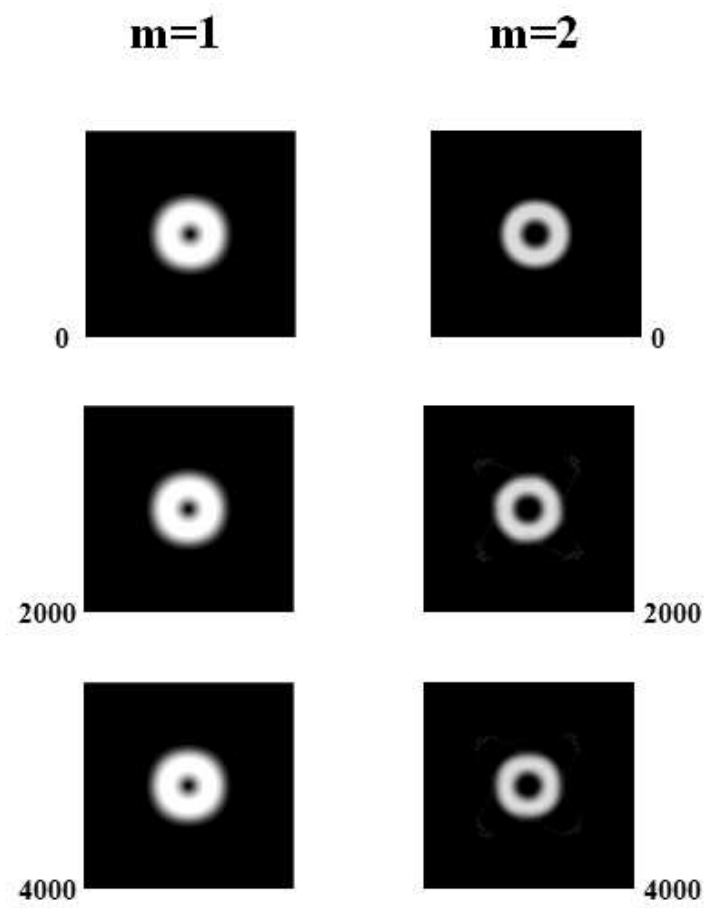

FIG. 9: Vortex dynamics (for different time-moments) when $\lambda=0.2$, the vortex is robust towards perturbations; the left panel is for $m=1, A_{\max }=1.386$ while the right panel is for $m=2, A_{m}=$ 1.3729 . 